\title{
Performance and fouling analysis of an ultrafiltration membrane for lecithin separation from isopropanol- oleic acid-lecithin mixtures
}

\author{
Nita Aryanti ${ }^{\mathrm{a}, \mathrm{b}, *}$, Diah H. Wardhani ${ }^{\mathrm{a}}$, Tutuk D. Kusworo ${ }^{\mathrm{a}, \mathrm{b}}$, Aininu Nafiunisa ${ }^{\mathrm{a}}$ \\ a Chemical Engineering Department, Diponegoro University, Tembalang, Semarang 50275 Indonesia \\ b Membrane Research Centre (MeR-C), Diponegoro University, Tembalang, Semarang 50275 Indonesia
}

*Corresponding author, e-mail: nita.aryanti@che.undip.ac.id

Received 30 May 2019

Accepted 31 Dec 2019

\begin{abstract}
Crude palm oil (CPO) can be processed into various types of derivative products such as cooking oil, margarine, ice cream, and soap. To obtain good quality CPO, physical and chemical purification methods are often applied. Ultrafiltration (UF) membranes are an alternative method to purify CPO. However, fouling is a major limitation in the application of UF membrane technology. The most common sources of fouling in the purification of CPO are phospholipids and fatty acids. In this research, fouling phenomena are investigated to understand the relevant fouling mechanism of UF membranes. This study aims to evaluate the performance of a UF membrane for separating isopropanol-oleic acid-lecithin feed solutions. UF performance is evaluated based on the flux and rejection of phospholipids and free fatty acid. The fouling was identified by the value of relative flux reduction, percent of fouling and the membrane morphology after filtration. This research confirms that addition of oleic acid results in a significant decline in flux. In addition, cake/gel layer blocking is established to be the blocking mechanism of the UF process.
\end{abstract}

KEYWORDS: ultrafiltration, degumming, oil, fouling, Hermiar's model

\section{INTRODUCTION}

Indonesia is one of the largest palm oil producers in the world; in 2016, for example, the country produced 32 million tons of oil. Crude palm oil (CPO) is a common processed palm oil product. Approximately 4.50 million tons of CPO are processed for domestic palm cooking oil, while 6.87 million tons are exported in the form of CPO processed products. Processing the CPO into palm cooking oil is inseparable because the CPO contains nutrition such as Vitamin A (carotene), Vitamin E (Tocopherols and Tocotrienols) and $\beta$-carotene, $\alpha$-carotene [1]. An appropriate $\mathrm{CPO}$ processing method is thus required to produce good quality palm cooking oil. Several methods such as chemical separation, enzymatic separation, and membrane technology have been implemented to enable CPO processing. As each technique presents unique advantages and disadvantages, careful consideration is needed to select a suitable method. Chemical separation causes a considerable amount of oil and nutritive loss. It also requires extensive energy, water, and chemical consumption and produces vast amounts of liquid waste [2].

As an alternative to existing methods, ultrafil- tration (UF) membrane technology has been introduced to purify or degum CPO. This process utilizes membranes to separate gum from the vegetable oil. According to Lindau and Jönsson [3], membrane separation is mainly conducted based on molecule size and uses pressure as the driving force. Components are separated according to shape, weight, or particle size of molecules. Interactions between particle molecules and the membrane material influence the performance of UF membrane technology [4].

One of the major challenges in the application of UF membranes for degumming crude oil is the occurrence of fouling. Research on fouling during crude oil purification has been carried out by Pagliero et al [5], who clarified that the phenomenon may occur due to the accumulation and deposition of feed molecules on the membrane. Fouling can take place on the membrane surface and inside membrane pores. The degree of fouling resistance can be calculated by modeling. Determination of fouling resistance in ultrafiltration membrane was performed according to fouling layer resistance model [6] and has been developed for evaluation of organic gel fouling re- 
sistance [7] and fouling resistance due to proteinsaccharide [8] in ultrafiltration. In addition, Mah et al [9] determined irreversible fouling resistance $\left(R_{\text {ir }}\right)$ for palm oil-oleic acid-glycerin based on a resistance-in-series model through rearragement of Darcy's equation. Fouling is present in hydrophobic membranes and low-molecular weight feeds such as fatty acids [10]. Fatty acid molecules tend to be retained and adsorbed on the membrane surface, causing pore blockage. Fouling due to adsorption of fatty acids has significant effect on membrane performance, then studies focusing on membrane fouling phenomena are urgently required.

Previous studies on oil refining used membranes to refine soybean oil [11], crude sunflower oil [5], and CPO [1]. Another study attempted to separate phospholipids from residual palm fiber oil/hexane micelles, and high phospholipid retention rates in the range of $81-95 \%$ were reported [12]. However, analysis of the fouling mechanism is infrequently studied. Determining the correct blocking mechanism will lead to a better understanding of fouling prevention and potentially overcome the fouling problem. Membrane fouling can be analyzed in terms of models and mathematical calculations.

In this study, degumming of palm oil was conducted with emphasis on membrane performance, fouling phenomena, and the mechanism of fouling. The feed used is an approximate model of CPO consisting of isopropanol, oleic acid, and lecithin. This study aims to evaluate the performance of UF for degumming of crude oil by using an isopropanololeic acid-lecithin solution as a feed solution. Investigation of the fouling phenomena is also conducted to understand the relevant fouling mechanism.

\section{MATERIALS AND METHODS}

The main raw materials used in this experiment were isopropanol (Merck) as a solvent, oleic acid, and lecithin as a model phospholipid. The UF membrane was a laboratory-made polyethersulfone (PES) flat-sheet membrane. The PEG material was Veradel PESU 3100P (Solvay, Singapore). The membrane was prepared via a non-solvent-induced phase separation method with PEG as the additive and $N$-methyl-2-pyrrolidone as the solvent. The prepared PES membrane had a Molecular Weight Cutoff (MWCO) of $25 \mathrm{kDa}$, contact angle of $63.63^{\circ}$, water permeability of $42.77 \mathrm{l} / \mathrm{m}^{2} . \mathrm{h}$, and isopropanol permeability of $63.58 \mathrm{l} / \mathrm{m}^{2}$.h. The characteristics of this membrane were detailed in a previous study by Aryanti et al [13].

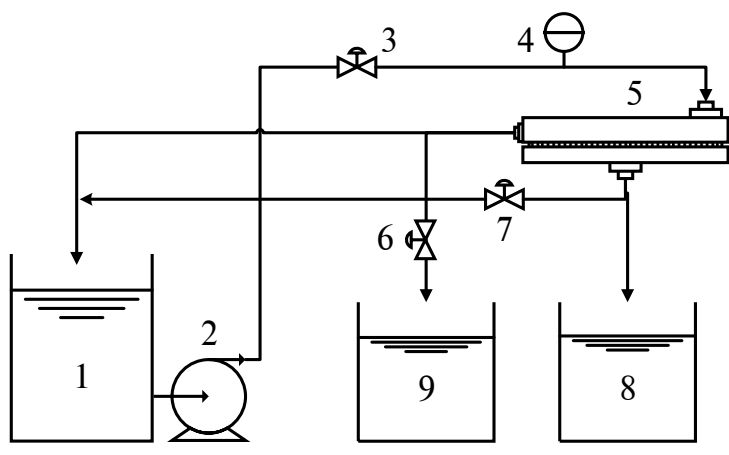

Fig. 1 Schematic of an ultrafiltration cell with total recycling operation: (1) feed tank, (2) feed pump, (3) feed valve, (4) pressure indicator, (5) ultrafiltration membrane, (6) retentate valve, (7) permeate valve, (8) permeate tank, and (9) retentate tank.

\section{Evaluation of UF membrane performance}

UF performance was examined by using a laboratory-made cell filtration unit based on the total recycling model, as illustrated in Fig. 1. The cell filtration unit was equipped with a centrifugal pump and stainless steel UF housing. The total recycling model involved returning the permeate and retentate flow back to the feed tank to maintain an equivalent concentration during the process. All experimental runs were conducted at room temperature $\left(25 \pm 2^{\circ} \mathrm{C}\right)$. Before starting the experiments, the membranes were first compacted by filtering water through them at a pressure of 1 bar for $60 \mathrm{~min}$. For each run, a new circular membrane sheet with an effective area of $13.85 \mathrm{~cm}^{2}$ was used.

A micellar solution was prepared by mixing isopropanol, oleic acid, and lecithin $(5 \% \mathrm{w} / \mathrm{w})$ as the model foulant at various concentrations of oleic acid ( $40 \%, 43 \%$, and $45 \% \mathrm{w} / \mathrm{w}$ of the solution). The filtration cell was operated at 1 bar for $120 \mathrm{~min}$, prior to its return to the feed tank; the permeate was collected every $5 \mathrm{~min}$ to determine the flux and concentration of phospholipids/fatty acids. The feed tank was equipped with a magnetic stirrer for homogenization of oil micelles. Membrane performance was evaluated in terms of permeate flux and phospholipid/free fatty acid (FFA) rejection. Permeate fluxes $(J)$ were determined by weighing the volume of the permeate collected at $5 \mathrm{~min}$ intervals for $120 \mathrm{~min}$ and calculated using $J=W / A t$, where $W$ represents the total weight of the permeate, $A$ is the membrane area, and $t$ is the time interval.

Rejection of phospholipids and FFAs $(R)$ was de- 
Table 1 Linearization of the Hermia's model.

\begin{tabular}{lll}
\hline Pore blocking model & Equation & Physical concept \\
\hline Complete & $\ln J=\ln J_{0}-K_{\mathrm{c}} t$ & Surface deposit \\
Intermediate & $\frac{1}{J}=\frac{1}{J_{0}}+K_{\mathrm{i}} t$ & Pore constriction \\
Standard & $\frac{1}{\sqrt{J}}=\frac{1}{\sqrt{J_{0}}}+K_{\mathrm{s}} t$ & Pore blocking + \\
Gel/cake formation & $\frac{1}{J^{2}}=\frac{1}{J_{0}^{2}}+K_{\mathrm{cf}} t$ & Pore blocking \\
\hline
\end{tabular}

termined on the basis of the concentration of phospholipids/FFA in the feed $\left(C_{\mathrm{f}}\right)$ and permeate $\left(C_{\mathrm{p}}\right)$ according to equation

$$
R=\frac{C_{\mathrm{f}}-C_{\mathrm{p}}}{C_{\mathrm{f}}} .
$$

\section{Characterization of refined oil and permeate}

After filtration process, phospholipid and Free Fatty Acid (FFA) content in the permeate were analyzed. Phospholipids are expressed in terms of total phosphorus and were analyzed according to the AOAC Ca 12-55 Method. Determination of FFA was performed via the acid-base titration method [14]. All of the analysis was conducted without any pretreatment or $\mathrm{pH}$ adjustment.

\section{Statistical data analysis}

All of the analysis was conducted in triplicate, to avoid any miss-measurement or error and to make sure that the obtained data were valid. The presented data are the average of the three-time analysis along with the standard deviation. The relative standard deviation (RSD) was determined to be under $5 \%$. The graphical plots were performed using Microsoft Excel 2010 with the same statistical judgement.

\section{Blocking mechanism}

The blocking mechanism of isopropanol-oleic acidlecithin UF was studied according to Hermia's model. This model has been previously applied to evaluate the fouling mechanism during UF of a dye wastewater model [15], UF of dye solution [16], and separation of konjac glucomannan [17]. Hermia's model describes the mechanism of membrane fouling on the basis of several blocking filtration laws, which consist of complete pore blocking, standard pore blocking, intermediate pore blocking, and cake filtration. The blocking filtration law is expressed in terms of permeation volume and filtration time and was developed for dead-end filtration [13],

$$
\frac{\mathrm{d}^{2} t}{\mathrm{~d} V^{2}}=k\left(\frac{\mathrm{d} t}{\mathrm{~d} V}\right)^{n}
$$

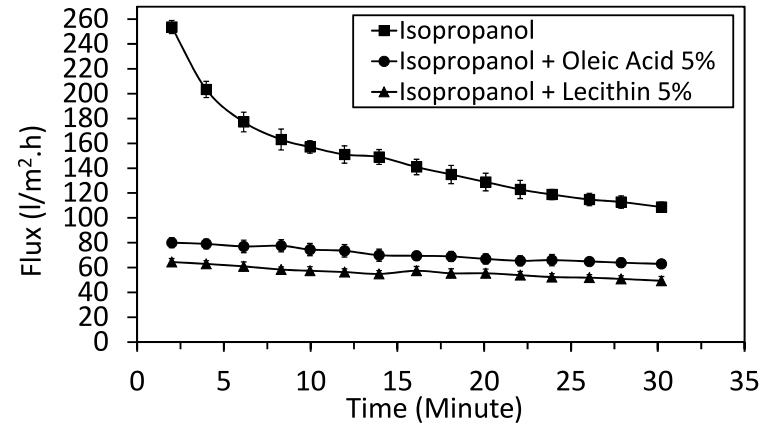

Fig. 2 Flux profiles for isopropanol (IPA), a mixture of IPA and oleic acid, and a mixture of IPA with lecithin.

where $t$ is the filtration time, $V$ is the permeate volume, $k$ is a constant, and $n$ is a value illustrating the different fouling mechanisms.

The values of $n$ describe complete blocking, intermediate blocking, standard blocking, and gel/cake filtration. Complete blocking is defined when $n=2$, intermediate blocking occurs when $n=$ 1 , standard blocking occurs when $n=1.5$, and cake layer formation occurs when $n=0$. In the complete blocking model, each solute molecule is assumed to participate in blocking the entrance of the membrane pores completely. In intermediate blocking, each solute molecule is assumed to stay on previously deposited solutes. Standard blocking considers the deposition of solute molecules to the internal pore wall. Cake layer formation occurs due to the accumulation of the solute on the membrane surface in the form of a cake [18]. Hermia's model was then linearized on the basis of the $n$ of each model in terms of permeate flux versus time, by fitting equations in Table 1.

\section{RESULTS}

\section{Effect of feed composition on the flux profiles}

Fig. 2 shows the performance of the UF membrane with various feed compositions in terms of flux profiles.

The figure reveals variations in fouling tendency according to the feed solution. The flux significantly declines when the feed contains isopropanol only. The large decline in the flux occurring at the beginning of the filtration operation compared with that at the end of the process indicates the occurrence of intermediate fouling. 


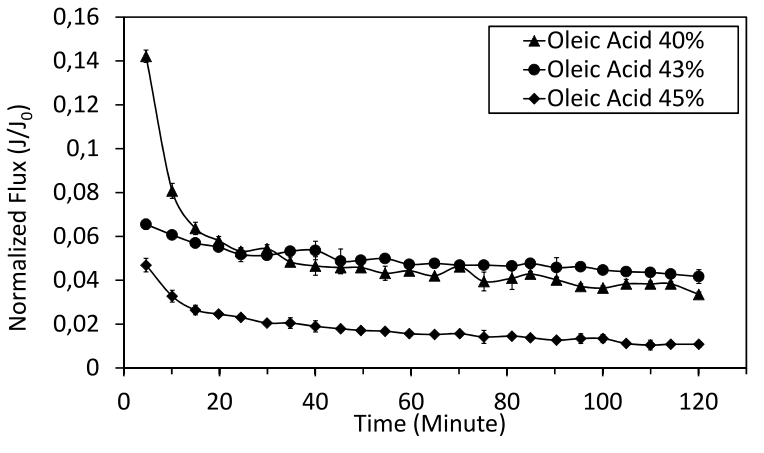

Fig. 3 Flux profiles of $5 \%(w / w)$ isopropanol-lecithin mixtures with $40 \%, 43 \%$, and $45 \%$ oleic acid.

Table 2 Phospholipid and FFA rejection rates.

\begin{tabular}{lcr}
\hline Oleic acid concentration & \% Phospolipid & \multicolumn{1}{c}{$\%$ FFA } \\
\hline $40 \%$ of oleic acid & $56.48 \pm 0.83$ & $8.16 \pm 0.25$ \\
$43 \%$ of oleic acid & $62.25 \pm 1.14$ & $10.57 \pm 0.64$ \\
$45 \%$ of oleic acid & $78.52 \pm 0.98$ & $16.39 \pm 0.54$ \\
\hline
\end{tabular}

Effect of different feed compositions on the flux profiles

The three feed solutions consisted of phospholipids in the same amount and various concentrations of oleic acid, i.e., 40\%, 43, and 45\%. Fig. 3 presents the flux profiles of these three solutions. The figure shows that the flux decreases with increasing addition of oleic acid.

\section{Effect of feed composition on phospholipid and FFA rejection rates}

Table 2 represents the rejection rates of phospholipid and FFA at various oleic acid concentrations. The rejection rates reveal membrane selectivity when removing a compound from the feed. According to the table, UF can reject larger amounts of phospholipids with higher concentrations of oleic acid. By contrast, rejection of FFA tends to be low at any oleic acid concentration.

\section{Blocking mechanism by Hermia's model}

Fig. 4 presents the linearized flux profiles according to Hermia's model, which comprises several

Table 3 Fouling parameters based on the Hermia's model.

\begin{tabular}{lcccc}
\hline & $n=2$ & $n=1.5$ & $n=1$ & $n=0$ \\
\hline Oleic acid 40\% & 0.6560 & 0.7447 & 0.8151 & 0.8948 \\
Oleic acid 43\% & 0.9355 & 0.9485 & 0.9586 & 0.9701 \\
Oleic acid 45\% & 0.8683 & 0.9172 & 0.9472 & 0.9632 \\
\hline
\end{tabular}

blocking models. Fitting of the experimental results to Hermia's model was conducted to understand the mechanism of membrane fouling during UF of isopropanol-lecithin-oleic acid. By plotting the linearized fitting data, the mechanism fouling could be identified.

According to the $R^{2}$ data, as shown in Table 3, the process of UF of the model solution containing refined CPO, isopropanol, lecithin, and oleic acid fits the cake formation model for each acid concentration. The membrane morphologies before and after UF are presented in Fig. 5. The membrane surface after UF is fully covered by a foulant. This finding confirms that the blocking mechanism during the UF process is cake/gel formation [9].

\section{DISCUSSION}

\section{Effect of feed composition on the flux profiles}

In general, the flux declines with increasing filtration time, and large declines in the flux are observed when isopropanol added with a model foulant (i.e., both lecithin and oleic acid) is used as a feed. Greater declines in the flux are also observed at the beginning of the filtration operation than at the end of the process. This phenomenon indicates a complete blocking mechanism. It was presumable that a great number of membrane pores are reduced significantly and blocking the fluid to move through the membrane. Similar conditions have been reported for the UF of soybean oil using a polymeric membrane [1].

The flux profiles of the model solution tend to be stable, and the flux of the feed with isopropanol only is higher than that of the model solution. Both model solutions show low flux due to the direct formation of a foulant/gel layer on the membrane surface. According to the flux profile, it was predicted that specific fouling resistance $(\alpha)$ in small fluxes of isopropranol and oleic acid as well as isopropanol and lecithin tend to remain constant indicating the fouling layer was invariable and the fouling thickness increased by deposition or mass addition [8]. On the other hand, at higher flux, there was a change in fouling layer structural due to an increase of fluid drag and associated compressive stress [8]. Further, based on specific fouling resistance, compressibility index representing compressible or incompressible fouling layers could be determined [7]. However, in the research, both specific fouling resistance and compressibility index were not able to be calculated since we did not have a record of pressure drop due to fouling $\left(\Delta P_{\mathrm{c}}\right)$ as a 

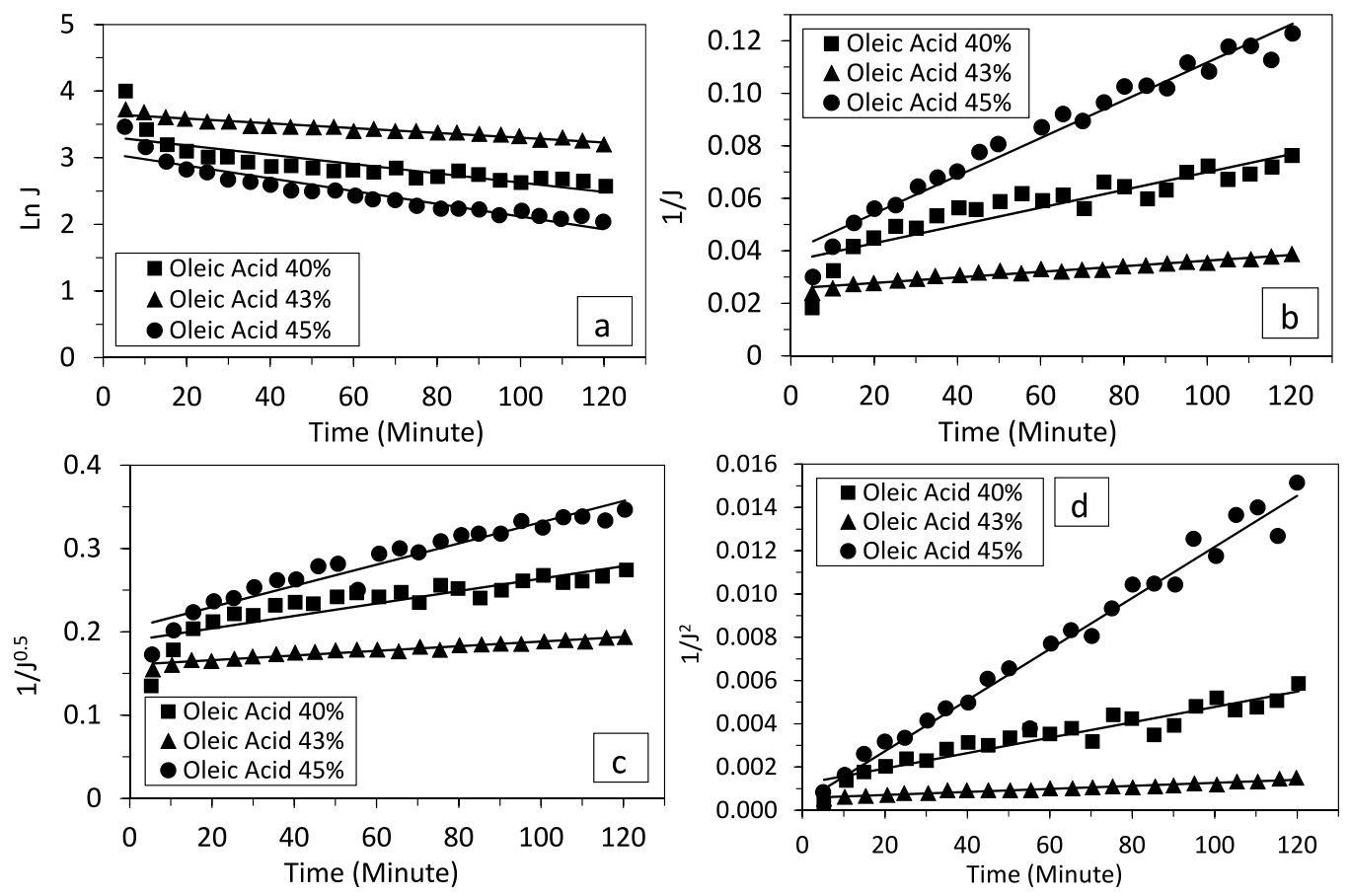

Fig. 4 Curve fitting of 5\% isopropanol-lecithin mixtures with various oleic acid concentrations to Hermia's model: (a) complete blocking, (b) intermediate blocking, (c) standard blocking, and (d) cake formation.
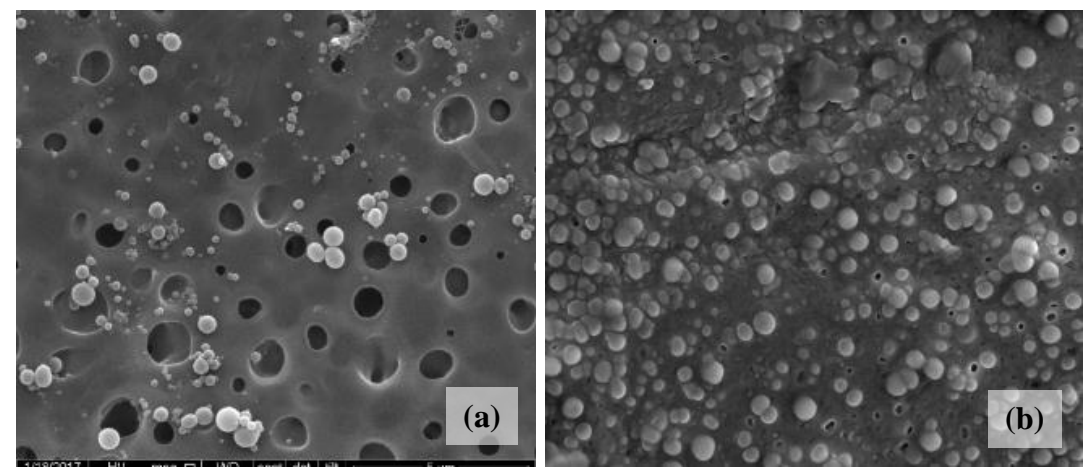

Fig. 5 Scanning electron micrographs of the membrane (a) before and (b) after UF at 43\% concentration of oleic acid.

function of time.

Both oleic acid and lecithin/phospholipid influence the formation of the deposited cake layer. At the same concentration, the component with the most influence on fouling is lecithin/phospholipid. Phospholipids are large amphiphilic molecules with both positive and negative charges, and their amphiphilic characteristics can maintain neutral properties. However, the distribution of negatively charged phospholipids, namely phosphatidyl choline, on the membrane surface can induce coagulation, which results in the formation of clots [19]. Moreover, the PES membrane is not a highly hy- drophobic membrane, and the long alkyl chains of the phospholipids could increase hydrophobic interactions with hydrophobic polymer membranes to form a stable layer [20]. Oleic acid has a double bond between molecules and is considered an unsaturated fatty acid (UFA). Furthermore, the acid has low hydrophobicity and could reduce the effect of layer formation, thereby inducing a higher flux than lecithin. 


\section{Effect of different feed compositions on the flux profiles}

Higher concentrations of oleic acid result in significantly decreased fluxes because oleic acid is a UFA with few hydrophobic properties. The mixture of UFAs with saturated fatty acids reduces the hydrophobic properties of the latter. As mentioned earlier, the PES membrane is partly hydrophobic with a contact angle of $63.63^{\circ}$, and the addition of oleic acid decreases the hydrophobic properties of the feed. As a result, the interaction between the feed and the membrane surface is reduced and their effect on the flux decreases.

Fig. 3 also shows that all of the profile fluxes decline over time due to the formation of a gel layer. This gel layer is often observed to be the result of concentration polarization on the surface of the membrane $[5,21,22]$. Concentration polarization refers to the increase in solute concentration on the membrane surface due to membrane interactions with the solute. This cause of concentration polarization is not only due to the hydrophobic interactions of phospholipids on the membrane but also oleic acid as a foulant. Thus, a layer is formed on the surface of the membrane and blocks flow through the membrane. Further processing leads to a formation of fouling on the membrane, resulting in a decrease in flux during the operation [23].

\section{Effect of feed composition on phospholipid and FFA rejection rates}

The dispersion of phospholipids in the nonpolar solvent, isopropanol, forms reverse micelle with an average weight greater than that of triglyceride [5]. The molecular weight of phospholipids increase up to $20 \mathrm{kDa}$, thus allowing phospholipids to be rejected by the membrane pore and the oil and the isopropanol goes through the membrane as permeate. The rejection of phospholipid also increases by the addition of oleic acid. This was due to the different hydrophobic properties of the feed with different concentrations of oleic acid. As explained before, the addition of oleic acid reduced the hydrophobic properties of the feed, and formed more unsaturated condition. This results in the more molecule of phospholipid to be retained by the membrane and increases the rejection value. In contrast, the rejection of FFA shows a low value, with the highest rejection only $16.39 \%$. FFA has a molecular weight of $300 \mathrm{Da}$, which is smaller than the membrane pore $[18,24]$. The low value of FFA rejection also confirms a low selectivity of the membrane. In this study, the membrane is only selective to reject the phospholipid with the highest rejection value of $78.52 \%$.

\section{Blocking mechanism by Hermia's model}

In the cake formation model, particles are rejected by the membrane, and a cake layer builds over the membrane surface. Fouling is formed because of the reverse micelles of the phospholipid, which have a molecular weight larger than the MWCO of the membrane, thus causing them to be retained on the membrane surface. Interactions between the membrane surface and foulants also promote the formation of an irreversible cake layer. Moreover, the use of a hydrophobic PES membrane and the hydrophobic properties of the phospholipid helps form a cake foulant on the surface of the membrane.

\section{CONCLUSION}

The occurrence of fouling is influenced not only by the non-homogenous pore size of membranes and the particle size of the solute in the feed but also by interactions between membranes and components, the concentration of phospholipids, and membrane wettability. Phospholipids are the most important factor influencing fouling during purification of the CPO model using UF membranes. Increasing the concentration of oleic acid in the feed could reduce the hydrophobic properties of the system and inhibit the interaction of membrane molecules.

Acknowledgements: Authors thank Eko N. Widodo and Ridwan R. Ardiyansyah for their assistances. NA acknowledges Directorate of Research and Community Service, Ministry of Research, Technology and Higher Education, The Republic of Indonesia for financial support through Fundamental Research Grant No. 10145/UN7.P4.3/PP/2018.

\section{REFERENCES}

1. Arora S, Manjula S, Krishna AG, Subramanian R (2006) Membrane processing of crude palm oil. Desalination 191, 454-466.

2. de Souza MP, Petrus JCC, Gonçalves LAG, Viotto LA (2008) Degumming of corn oil/hexane miscella using a ceramic membrane. J Food Eng 86, 557-564.

3. Lindau J, Jönsson A-S (1999) Adsorptive fouling of modified and unmodified commercial polymeric ultrafiltration membranes. J Memb Sci 160, 65-76.

4. Doshi K, Trivedi Y, Ray P, Singh PS (2019) Degumming of crude vegetable oil by membrane separation: Probing structure-performance and stability of PVDF membranes. Sep Sci Technol 54, 360-369. 
5. Pagliero C, Mattea M, Ochoa N, Marchese J (2007) Fouling of polymeric membranes during degumming of crude sunflower and soybean oil. J Food Eng 78, 194-197.

6. Sioutopoulos DC, Karabelas AJ (2012) Correlation of organic fouling resistances in RO and UF membrane filtration under constant flux and constant pressure. $J$ Memb Sci 407-408, 34-46.

7. Sioutopoulos DC, Karabelas AJ (2016) Evolution of organic gel fouling resistance in constant pressure and constant flux dead-end ultrafiltration: Differences and similarities. J Memb Sci 511, 265-277.

8. Sioutopoulos D, Karabelas A, Mappas V (2019) Membrane fouling due to protein-polysaccharide mixtures in dead-end ultrafiltration; the effect of permeation flux on fouling resistance. Membranes 9, ID 21.

9. Mah SK, Chuah CK, Lee WC, Chai SP (2012) Ultrafiltration of palm oil-oleic acid-glycerin solutions: Fouling mechanism identification, fouling mechanism analysis and membrane characterizations. Sep Purif Technol 98, 419-431.

10. Mohammad AW, Amin INHM (2014) Fouling of ultrafiltration membrane during adsorption of long chain fatty acid in glycerine solutions. Sains Malaysiana 42, 827-836.

11. Ochoa N, Pagliero C, Marchese J, Mattea M (2001) Ultrafiltration of vegetable oils: Degumming by polymeric membranes. Sep Purif Technol 22, 417-422.

12. Majid RA, Mohammad AW, May CY (2013) Performance of polymeric membranes for phospholipid removal from residual palm fibre oil/hexane miscella. J Oil Palm Res 25, 253-264.

13. Aryanti N, Wardhani DH, Nafiunisa A (2018) Ultrafiltration membrane for degumming of crude palm Oil-isopropanol mixture. Chem Biochem Eng Q 32, 325-334.

14. Lai SO, Heng SL, Chong KC, Lau WJ (2016) Deacidification of palm oil using solvent extraction integrated with membrane technology. J Teknologi 78, 69-74.

15. Aryanti N, Sandria FKI, Wardhani DH (2017)
Blocking mechanism of ultrafiltration and micellarenhanced ultrafiltration membrane for dye removal from model waste water. Adv Sci Lett 23, 2598-2600.

16. Aryanti N, Sandria FKI, Putriadi RH, Wardhani DH (2017) Evaluation of micellar-enhanced ultrafiltration (MEUF) membrane for dye removal of synthetic remazol dye wastewater. Eng $J$ 21, 23-35.

17. Aryanti N, Wardhani DH, Supandi S (2016) Flux profiles and mathematical modeling of fouling mechanism for ultrafiltration of Konjac glucomannan. Chem Chem Eng Biotechnol Food Ind 17, 125-137.

18. Amin INHM, Mohammad AW, Markom M, Choe PL, Hilal N (2010) Analysis of deposition mechanism during ultrafiltration of glycerin-rich solutions. Desalination 261, 313-320.

19. Lamas DL, Constenla T, Raab D (2016) Effect of degumming process on physicochemical properties of sunflower oil. Biocatal Agric Biotechnol 6, 138-143.

20. Elliott JT, Burden DL, Woodward JT, Sehgal A, Douglas JF (2003) Phospholipid monolayers supported on spun cast polystyrene films. Langmuir 19, 2275-2283.

21. Penha FM, Rezzadori K, Proner MC, Zin G, Fogaça LA, Petrus JCC, de Oleviera JV, Luccio MD (2015) Evaluation of permeation of macauba oil and n-hexane mixtures through polymeric commercial membranes subjected to different pre-treatments. J Food Eng 155, 79-86.

22. Kim I-C, Kim J-H, Lee K-H, Tak T-M (2002) Phospholipids separation (degumming) from crude vegetable oil by polyimide ultrafiltration membrane. J Memb Sci 205, 113-123.

23. Sehn GA, Gonçalves LA, Ming CC (2016) Ultrafiltration-based degumming of crude rice bran oil using a polymer membrane. Grasas Aceites 67, ID e120.

24. Firman L, Ochoa NA, Marchese J, Pagliero C (2017) Simultaneous improvement in solvent permeability and deacidification of soybean oil by nanofiltration. J Food Sci Technol 54, 398-407. 